\title{
Complex Dynamical Network Control for Trajectory Tracking Using Delayed Recurrent Neural Networks
}

\author{
Jose P. Perez, Joel Perez Padron, Angel Flores Hemandez, and Santiago Arroyo \\ Facultad de Ciencias Fisico Matematicas, Universidad Autonoma de Nuevo Leon (UANL), 66451 San Nicolás de los Garza, \\ NL, Mexico \\ Correspondence should be addressed to Jose P. Perez; josepazp@gmail.com
}

Received 21 March 2013; Accepted 12 September 2013; Published 2 January 2014

Academic Editor: Chuandong Li

Copyright (C) 2014 Jose P. Perez et al. This is an open access article distributed under the Creative Commons Attribution License, which permits unrestricted use, distribution, and reproduction in any medium, provided the original work is properly cited.

In this paper, the problem of trajectory tracking is studied. Based on the V-stability and Lyapunov theory, a control law that achieves the global asymptotic stability of the tracking error between a delayed recurrent neural network and a complex dynamical network is obtained. To illustrate the analytic results, we present a tracking simulation of a dynamical network with each node being just one Lorenz's dynamical system and three identical Chen's dynamical systems.

\section{Introduction}

The analysis and control of complex behavior in complex networks, which consist of dynamical nodes, have become a point of great interest in the recent studies, [1-3]. The complexity in networks comes not only from their structure and dynamics but also from their topology, which often affects their function.

Recurrent neural networks have been widely used in the fields of optimization, pattern recognition, signal processing and control systems, among others. They have to be designed in such a way that there is one equilibrium point that is globally asymptotically stable. In biological and artificial neural networks, time delays arise in the processing of information storage and transmission. Also, it is known that these delays can create oscillatory or even unstable trajectories, [4]. Trajectory tracking is a very interesting problem in the field of theory of systems control; it allows the implementation of important tasks for automatic control such as: high speed target recognition and tracking, real-time visual inspection, and recognition of context sensitive and moving scenes, among others. We present the results of the design of a control law that guarantee the tracking of general complex dynamical networks.

\section{Mathematical Models}

2.1. General Complex Dynamical Networks. Consider a network consisting of $N$ linearly and diffusively coupled nodes, with each node being an $n$-dimensional dynamical system, described by

$$
\dot{x}_{i}=f_{i}\left(x_{i}\right)+\sum_{\substack{j=1 \\ j \neq i}}^{N} c_{i j} a_{i j} \Gamma\left(x_{j}-x_{i}\right), \quad i=1,2, \ldots, N,
$$

where $x_{i}=\left(x_{i 1}, x_{i 2}, \ldots, x_{i n}\right)^{T} \in \mathbb{R}^{n}$ are the state vectors of the node $i, f_{i}: \mathbb{R}^{n} \mapsto \mathbb{R}^{n}$ represents the self-dynamics of the node $i$, and the constants $c_{i j}>0$ are the coupling strengths between node $i$ and node $j$, with $i, j=1,2, \ldots, N$. $\Gamma=\left(\tau_{i j}\right) \in \mathbb{R}^{n \times n}$ is a constant internal matrix that describes the way of linking the components in each pair of connected node vectors $\left(x_{j}-\right.$ $\left.x_{i}\right)$ : this means that for some pairs $(i, j)$ with $1 \leq i, j \leq n$ and $\tau_{i j} \neq 0$, the two coupled nodes are linked through their $i$ th and $j$ th substate variables, respectively, while the coupling matrix $A=\left(a_{i j}\right) \in \mathbb{R}^{N \times N}$ denotes the coupling configuration of the entire network: this means that if there is a connection between node $i$ and node $j(i \neq j)$, then $a_{i j}=a_{j i}=1$; otherwise, $a_{i j}=a_{j i}=0$. 
2.2. Delayed Recurrent Neural Networks. Consider a delayed recurrent neural network in the following form:

$$
\begin{gathered}
\dot{x}_{n_{i}}=A_{n_{i}} x_{n_{i}}+W_{n_{i}} \sigma\left(x_{n_{i}}(t-\tau)\right)+u_{n_{i}} \\
+\sum_{\substack{j=1 \\
j \neq i}}^{N} c_{n_{i} n_{j}} a_{n_{i} n_{j}} \Gamma\left(x_{n_{j}}-x_{n_{i}}\right), \\
i=1,2, \ldots, N,
\end{gathered}
$$

where $\tau$ is the fixed known time delay $[5,6], x_{n_{i}}=\left(x_{n_{i} 1}\right.$, $\left.x_{n_{i} 2}, \ldots, x_{n_{i} n}\right)^{T} \in \mathbb{R}^{n}$ is the state vector of the neural network $i$, $u_{n_{i}} \in \mathbb{R}^{n}$ is the input of the neural network $i, A_{n_{i}}=-\lambda_{n_{i}} I_{n \times n}$, $i=1,2, \ldots, N$, is the state feedback matrix, with $\lambda_{n_{i}}$ being a positive constant, $W_{n_{i}} \in \mathbb{R}^{n \times n}$ is the connection weight matrix with $i=1,2, \ldots, N$, and $\sigma(\cdot) \in \mathbb{R}^{n}$ is a Lipschitz sigmoid vector function $[7,8]$, such that $\sigma\left(x_{n_{i}}\right)=0$ only at $x_{n_{i}}=0$, with Lipschitz constant $L_{\sigma_{i}}, i=1,2, \ldots, N$, and neuron activation functions $\sigma_{i}(\cdot)=\tanh (\cdot), i=1,2, \ldots, n$.

\section{Trajectory Tracking}

The objective is to develop a control law such that the $i$ th neural network (2) tracks the trajectory of the $i$ th dynamical system (1). We define the tracking error as $e_{i}=x_{n_{i}}-x_{i}$, $i=1,2, \ldots, N$, whose derivative with respect to time is

$$
\dot{e}_{i}=\dot{x}_{n_{i}}-\dot{x}_{i}, \quad i=1,2, \ldots, N .
$$

Substituting (1) and (2) in (3), we obtain

$$
\begin{aligned}
\dot{e}_{i}= & A_{n_{i}} x_{n_{i}}+W_{n_{i}} \sigma\left(x_{n_{i}}(t-\tau)\right)+u_{n_{i}}-f_{i}\left(x_{i}\right) \\
& +\sum_{\substack{j=1 \\
j \neq i}}^{N} c_{n_{i} n_{j}} a_{n_{i} n_{j}} \Gamma\left(x_{n_{j}}-x_{n_{i}}\right) \\
& -\sum_{\substack{j=1 \\
j \neq i}}^{N} c_{i j} a_{i j} \Gamma\left(x_{j}-x_{i}\right), \quad i=1,2, \ldots, N .
\end{aligned}
$$

Adding and subtracting $W_{n_{i}} \sigma\left(x_{i}(t-\tau)\right), \alpha_{i}(t), i=1,2, \ldots, N$, to (4), where $\alpha_{i}$ will be determined below, and considering that $x_{n_{i}}=e_{i}+x_{i}, i=1,2, \ldots, N$, then

$$
\begin{aligned}
\dot{e}_{i}= & W_{n_{i}}\left(\sigma\left(x_{n_{i}}(t-\tau)\right)-\sigma\left(x_{i}(t-\tau)\right)\right) \\
& +\left(u_{n_{i}}-\alpha_{i}(t)\right)+A_{n_{i}} e_{i} \\
& +\left(A_{n_{i}} x_{i}+W_{n_{i}} \sigma\left(x_{i}(t-\tau)\right)+\alpha_{i}(t)\right)-f_{i}\left(x_{i}\right) \\
& +\sum_{\substack{j=1 \\
j \neq i}}^{N} c_{n_{i} n_{j}} a_{n_{i} n_{j}} \Gamma\left(x_{n_{j}}-x_{n_{i}}\right) \\
& -\sum_{\substack{j=1 \\
j \neq i}}^{N} c_{i j} a_{i j} \Gamma\left(x_{j}-x_{i}\right), \quad i=1,2, \ldots, N .
\end{aligned}
$$

In order to guarantee that the $i$ th neural network (2) tracks the $i$ th reference trajectory (1), the following assumption has to be satisfied:

Assumption 1. There exist functions $\rho_{i}(t)$ and $\alpha_{i}(t), i=1$, $2, \ldots, N$, such that

$$
\begin{gathered}
\frac{d \rho_{i}(t)}{d t}=A_{n_{i}} \rho_{i}(t)+W_{n_{i}} \sigma\left(\rho_{i}(t)\right)+\alpha_{i}(t), \\
\rho_{i}(t)=x_{i}(t), \quad i=1,2, \ldots, N .
\end{gathered}
$$

Let us define

$$
\begin{gathered}
\tilde{u}_{n_{i}}=\left(u_{n_{i}}-\alpha_{i}(t)\right), \\
\phi_{\sigma}(t-\tau)=\sigma\left(x_{n_{i}}(t-\tau)\right)-\sigma\left(x_{i}(t-\tau)\right), \\
i=1,2, \ldots, N .
\end{gathered}
$$

Considering (6) and (7), equation (5) is reduced to

$$
\begin{aligned}
\dot{e}_{i}= & A_{n_{i}} e_{i}+W_{n_{i}} \phi_{\sigma}(t-\tau)+\widetilde{u}_{n_{i}} \\
& +\sum_{\substack{j=1 \\
j \neq i}}^{N} c_{n_{i} n_{j}} a_{n_{i} n_{j}} \Gamma\left(x_{n_{j}}-x_{n_{i}}\right) \\
& -\sum_{\substack{j=1 \\
j \neq i}}^{N} c_{i j} a_{i j} \Gamma\left(x_{j}-x_{i}\right), \quad i=1,2, \ldots, N .
\end{aligned}
$$

Writing the summations as

$$
\begin{aligned}
\sum_{\substack{j=1 \\
j \neq i}}^{N} c_{n_{i} n_{j}} a_{n_{i} n_{j}} \Gamma\left(x_{n_{j}}-x_{n_{i}}\right) & \left(\sum_{\substack{j=1 \\
j \neq i}}^{N} c_{n_{i} n_{j}} a_{n_{i} n_{j}} x_{n_{j}}-x_{n_{i}} \sum_{\substack{j=1 \\
j \neq i}}^{N} c_{n_{i} n_{j}} a_{n_{i} n_{j}}\right) \\
= & \Gamma \\
& \times \sum_{\substack{j=1 \\
j \neq i}}^{N} c_{i j} a_{i j} \Gamma\left(x_{j}-x_{i}\right){ }_{\substack{j=1 \\
j \neq i}}^{N} c_{i j} a_{i j} \\
= & \Gamma\left(\sum_{\substack{j=1 \\
j \neq i}}^{N} c_{i j} a_{i j} x_{j}-x_{i} \sum_{\substack{j \\
j}}^{N}, \ldots, N\right.
\end{aligned}
$$

and using that $c_{n_{i} n_{j}}=c_{i j}$ and $a_{n_{i} n_{j}}=a_{i j}$, then, using the equations above, (8) becomes

$$
\begin{array}{r}
\dot{e}_{i}=A_{n_{i}} e_{i}+W_{n_{i}} \phi_{\sigma}(t-\tau)+\tilde{u}_{n_{i}} \\
+\Gamma\left(\sum_{\substack{j=1 \\
j \neq i}}^{N} c_{i j} a_{i j} e_{j}-e_{i} \sum_{\substack{j=1 \\
j \neq i}}^{N} c_{i j} a_{i j}\right)
\end{array}
$$




$$
\begin{aligned}
= & A_{n_{i}} e_{i}+W_{n_{i}} \phi_{\sigma}(t-\tau)+\widetilde{u}_{n_{i}} \\
& +\sum_{\substack{j=1 \\
j \neq i}}^{N} c_{i j} a_{i j} \Gamma\left(e_{j}-e_{i}\right), \quad i=1,2, \ldots, N .
\end{aligned}
$$

It is clear that $e_{i}=0, i=1,2, \ldots, N$, is an equilibrium point of (10), when $\tilde{u}_{n_{i}}=0, i=1,2, \ldots, N$. In this way, the tracking problem can be restated as a global asymptotic stabilization problem for the system (10).

\section{Tracking Error Stabilization and Control Design}

In order to establish the convergence of (10) to $e_{i}=0$, $i=1,2, \ldots, N$, which ensures the desired tracking, first, we propose the following Lyapunov function:

$$
\begin{aligned}
& V_{N}(e)= \sum_{i=1}^{N} V\left(e_{i}\right) \\
&= \sum_{i=1}^{N}\left(\frac{1}{2}\left\|e_{i}\right\|^{2}+\int_{t-\tau}^{t}\left(\phi_{\sigma}^{T}(s) W_{n_{i}}^{T} W_{n_{i}} \phi_{\sigma}(s)\right) d s\right), \\
& e=\left(e_{1}^{T}, \ldots, e_{N}^{T}\right)^{T} .
\end{aligned}
$$

The time derivative of (11), along the trajectories of (10), is

$$
\begin{aligned}
& \dot{V}_{N}(e)=\frac{\partial V_{N}(e)}{\partial e} \dot{e}=\sum_{i=1}^{N} \frac{\partial V_{N}(e)}{\partial e_{i}} \dot{e}_{i} \\
& =\sum_{i=1}^{N}\left(\phi_{\sigma}^{T}(t) W_{n_{i}}^{T} W_{n_{i}} \phi_{\sigma}(t)\right. \\
& -\phi_{\sigma}^{T}(t-\tau) W_{n_{i}}^{T} W_{n_{i}} \phi_{\sigma}(t-\tau) \\
& +e_{i}^{T}\left(A_{n_{i}} e_{i}+W_{n_{i}} \phi_{\sigma}(t-\tau)+\widetilde{u}_{n_{i}}\right. \\
& \left.\left.+\sum_{\substack{j=1 \\
j \neq i}}^{N} c_{i j} a_{i j} \Gamma\left(e_{j}-e_{i}\right)\right)\right)
\end{aligned}
$$

Reformulating (12), we get

$$
\begin{aligned}
\dot{V}_{N}(e)=\sum_{i=1}^{N}( & -\lambda_{n_{i}}\left\|e_{i}\right\|^{2}+e_{i}^{T} W_{n_{i}} \phi_{\sigma}(t-\tau) \\
& +e_{i}^{T} \tilde{u}_{n_{i}}+\sum_{\substack{j=1 \\
j \neq i}}^{N} c_{i j} a_{i j} e_{i}^{T} \Gamma\left(e_{j}-e_{i}\right) \\
& +\phi_{\sigma}^{T}(t) W_{n_{i}}^{T} W_{n_{i}} \phi_{\sigma}(t) \\
& \left.-\phi_{\sigma}^{T}(t-\tau) W_{n_{i}}^{T} W_{n_{i}} \phi_{\sigma}(t-\tau)\right) .
\end{aligned}
$$

Next, let us consider the following inequality, proved in [9, 10]:

$$
X^{T} Y+Y^{T} X \leq X^{T} \Lambda X+Y^{T} \Lambda^{-1} Y,
$$

which holds for all matrices $X, Y \in \mathbb{R}^{n \times k}$ and $\Lambda \in \mathbb{R}^{n \times n}$ with $\Lambda=\Lambda^{T}>0$. Applying (14) with $\Lambda=I_{n \times n}$ to the term $e_{i}^{T} W_{n_{i}} \phi_{\sigma}(t-\tau), i=1,2, \ldots, N$, we get

$$
\begin{aligned}
& e_{i}^{T} W_{n_{i}} \phi_{\sigma}(t-\tau) \\
& \leq \frac{1}{2} e_{i}^{T} e_{i}+\frac{1}{2} \phi_{\sigma}^{T}(t-\tau) W_{n_{i}}^{T} W_{n_{i}} \phi_{\sigma}(t-\tau) \\
& \times \frac{1}{2}\left\|e_{i}\right\|^{2}+\frac{1}{2} \phi_{\sigma}^{T}(t-\tau) W_{n_{i}}^{T} W_{n_{i}} \phi_{\sigma}(t-\tau), \\
& i=1,2, \ldots, N .
\end{aligned}
$$

Then, we have that

$$
\begin{aligned}
\dot{V}_{N}(e) \leq \sum_{i=1}^{N}\left(-\lambda_{n_{i}}\left\|e_{i}\right\|^{2}+\frac{1}{2}\left\|e_{i}\right\|^{2}\right. \\
+\frac{1}{2} \phi_{\sigma}^{T}(t-\tau) W_{n_{i}}^{T} W_{n_{i}} \phi_{\sigma}(t-\tau) \\
+e_{i}^{T} \widetilde{u}_{n_{i}}+\sum_{\substack{j=1 \\
j \neq i}}^{N} c_{i j} a_{i j} e_{i}^{T} \Gamma\left(e_{j}-e_{i}\right) \\
+\phi_{\sigma}^{T}(t) W_{n_{i}}^{T} W_{n_{i}} \phi_{\sigma}(t) \\
\left.-\phi_{\sigma}^{T}(t-\tau) W_{n_{i}}^{T} W_{n_{i}} \phi_{\sigma}(t-\tau)\right) .
\end{aligned}
$$

By simplifying (16), we obtain

$$
\begin{aligned}
& \dot{V}_{N}(e) \leq \sum_{i=1}^{N}\left(-\lambda_{n_{i}}\left\|e_{i}\right\|^{2}+\frac{1}{2}\left\|e_{i}\right\|^{2}\right. \\
& -\frac{1}{2} \phi_{\sigma}^{T}(t-\tau) W_{n_{i}}^{T} W_{n_{i}} \phi_{\sigma}(t-\tau)+e_{i}^{T} \tilde{u}_{n_{i}} \\
& +\sum_{\substack{j=1 \\
j \neq i}}^{N} c_{i j} a_{i j} e_{i}^{T} \Gamma\left(e_{j}-e_{i}\right) \\
& \left.+\phi_{\sigma}^{T}(t) W_{n_{i}}^{T} W_{n_{i}} \phi_{\sigma}(t)\right) \\
& \leq \sum_{i=1}^{N}\left(-\lambda_{n_{i}}\left\|e_{i}\right\|^{2}+\frac{1}{2}\left\|e_{i}\right\|^{2}\right. \\
& +\phi_{\sigma}^{T}(t) W_{n_{i}}^{T} W_{n_{i}} \phi_{\sigma}(t)+e_{i}^{T} \widetilde{u}_{n_{i}} \\
& \left.+\sum_{\substack{j=1 \\
j \neq i}}^{N} c_{i j} a_{i j} e_{i}^{T} \Gamma\left(e_{j}-e_{i}\right)\right) .
\end{aligned}
$$


Since $\phi_{\sigma}$ is Lipschitz with Lipschitz constant $L_{\phi_{\sigma_{i}}}$ [7], then

$$
\begin{aligned}
\left\|\phi_{\sigma}(t)\right\| & =\left\|\sigma\left(x_{n_{i}}(t)\right)-\sigma\left(x_{i}(t)\right)\right\| \\
& \leq L_{\phi_{\sigma_{i}}}\left\|x_{n_{i}}(t)-x_{i}(t)\right\| \\
& =L_{\phi_{\sigma_{i}}}\left\|e_{i}(t)\right\|, \quad i=1,2, \ldots, N .
\end{aligned}
$$

Applying (18) to $\phi_{\sigma}^{T}(t) W_{n_{i}}^{T} W_{n_{i}} \phi_{\sigma}(t)$, we obtain

$$
\begin{aligned}
\phi_{\sigma}^{T}(t) W_{n_{i}}^{T} W_{n_{i}} \phi_{\sigma}(t) & \leq\left\|\phi_{\sigma}^{T}(t) W_{n_{i}}^{T} W_{n_{i}} \phi_{\sigma}(t)\right\| \\
& \leq\left(L_{\phi_{\sigma_{i}}}\right)^{2}\left\|W_{n_{i}}\right\|^{2}\left\|e_{i}\right\|^{2}, \quad i=1,2, \ldots, N .
\end{aligned}
$$

Now, (17) is reduced to

$$
\begin{aligned}
& \dot{V}_{N}(e) \leq \sum_{i=1}^{N}\left(-\lambda_{n_{i}}\left\|e_{i}\right\|^{2}+\frac{1}{2}\left\|e_{i}\right\|^{2}\right. \\
& +\left(L_{\phi_{\sigma_{i}}}\right)^{2}\left\|W_{n_{i}}\right\|^{2}\left\|e_{i}\right\|^{2}+e_{i}^{T} \widetilde{u}_{n_{i}} \\
& \left.+\sum_{\substack{j=1 \\
j \neq i}}^{N} c_{i j} a_{i j} e_{i}^{T} \Gamma\left(e_{j}-e_{i}\right)\right) \\
& =\sum_{i=1}^{N} e_{i}^{T}\left(-\lambda_{n_{i}} e_{i}-\sum_{\substack{j=1 \\
j \neq i}}^{N} c_{i j} a_{i j} \Gamma e_{i}\right. \\
& +\left(\frac{1}{2}+L_{\phi_{\sigma_{i}}}^{2}\left\|W_{n_{i}}\right\|^{2}\right) e_{i} \\
& \left.+\sum_{\substack{j=1 \\
j \neq i}}^{N} c_{i j} a_{i j} \Gamma e_{j}+\widetilde{u}_{n_{i}}\right) .
\end{aligned}
$$

We define $\tilde{u}_{n_{i}}=\widetilde{u}_{n_{i}}^{(1)}+\widetilde{u}_{n_{i}}^{(2)}, i=1,2, \ldots, N$, and then $(20)$ becomes

$$
\begin{aligned}
\dot{V}_{N}(e) \leq \sum_{i=1}^{N} e_{i}^{T}\left(-\lambda_{n_{i}} e_{i}-\sum_{\substack{j=1 \\
j \neq i}}^{N} c_{i j} a_{i j} \Gamma e_{i}\right. \\
+\left(\frac{1}{2}+L_{\phi_{\sigma_{i}}}^{2}\left\|W_{n_{i}}\right\|^{2}\right) e_{i}+\tilde{u}_{n_{i}}^{(1)} \\
\left.+\sum_{\substack{j=1 \\
j \neq i}}^{N} c_{i j} a_{i j} \Gamma e_{j}+\tilde{u}_{n_{i}}^{(2)}\right) .
\end{aligned}
$$

Now, we propose the use of the following control law:

$$
\begin{aligned}
\tilde{u}_{n_{i}}= & -\left(\frac{1}{2}+L_{\phi_{\sigma_{i}}}^{2}\left\|W_{n_{i}}\right\|^{2}\right) e_{i} \\
& -\sum_{\substack{j=1 \\
j \neq i}}^{N} c_{i j} a_{i j} \Gamma e_{j}, \quad i=1,2, \ldots, N .
\end{aligned}
$$

Then, $\dot{V}_{N}(e)<0$ for all $e \neq 0$. This means that the proposed control law (22) can globally and asymptotically stabilize the $i$ th error system (10), therefore ensuring the tracking of (1) by (2). Finally, the control action driving the recurrent neural networks is given by

$$
\begin{aligned}
u_{n_{i}}= & f_{i}\left(x_{i}\right)+\lambda_{n_{i}} x_{i}-W_{n_{i}} \sigma\left(x_{i}(t-\tau)\right) \\
& -\left(\frac{1}{2}+L_{\phi_{\sigma_{i}}}^{2}\left\|W_{n_{i}}\right\|^{2}\right) e_{i} \\
& -\sum_{\substack{j=1 \\
j \neq i}}^{N} c_{i j} a_{i j} \Gamma e_{j}, \quad i=1,2, \ldots, N .
\end{aligned}
$$

\section{Simulations}

In order to illustrate the applicability of the discussed results, we consider a dynamical network with just one Lorenz's node and three identical Chen's nodes. The single Lorenz system is described by

$$
\begin{gathered}
\left(\begin{array}{c}
\dot{x}_{1} \\
\dot{x}_{2} \\
\dot{x}_{3}
\end{array}\right)=\left(\begin{array}{c}
10 x_{2}-10 x_{1} \\
-x_{2}-x_{1} x_{2}+28 x_{1} \\
x_{1} x_{2}-\left(\frac{8}{3}\right) x_{3}
\end{array}\right) \\
x_{i}(0)=(10,0,10)^{T}, \quad i=1 .
\end{gathered}
$$

And the Chen's oscillator is described by

$$
\left(\begin{array}{c}
\dot{x}_{i 1} \\
\dot{x}_{i 2} \\
\dot{x}_{i 3}
\end{array}\right)
$$

$$
=\left(\begin{array}{c}
p_{1}\left(x_{i 2}-x_{i 1}\right)+\sum_{\substack{j=1 \\
j \neq i}}^{N} c_{i j} a_{i j}\left(x_{j 1}-x_{i 1}\right) \\
\left(p_{3}-p_{2}\right) x_{i 1}-x_{i 1} x_{i 3}+p_{3} x_{i 2}+\sum_{\substack{j=1 \\
j \neq i}}^{N} c_{i j} a_{i j}\left(x_{j 2}-x_{i 2}\right) \\
x_{i 1} x_{i 2}-p_{2} x_{i 3}+\sum_{\substack{j=1 \\
j \neq i}}^{N} c_{i j} a_{i j}\left(x_{j 3}-x_{i 3}\right) \\
x_{i}(0)=(-10,0,37)^{T}, \quad i=1,2,3,4 .
\end{array}\right)
$$




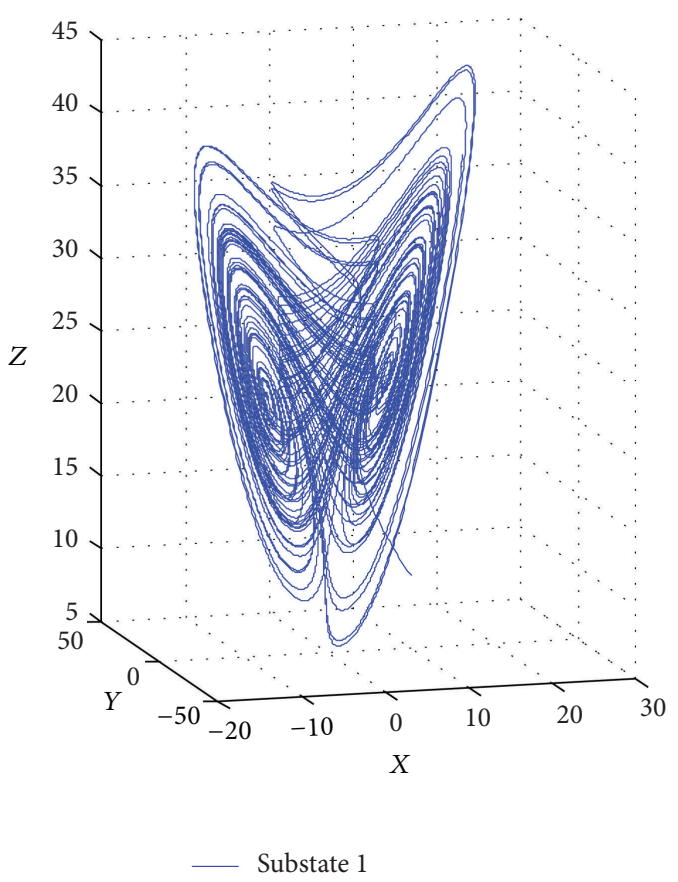

FIGURE 1: Substate of Lorentz's attractor with initial condition $X_{1}(0)=(10 ; 0 ; 10)^{T}$.

If the system parameters are selected as $p_{1}=35, p_{2}=3$, and $p_{3}=28$, then the Lorenz's system and Chen's system are shown in Figures 1 and 2, respectively. In this set of system parameters, one unstable equilibrium point of the oscillator (25) is $x=(7.9373,7.9373,21)^{T}$ [11].

Suppose that each pair of two connected Lorenz and Chen's oscillators are linked together through their identical substate variables; that is, $\Gamma=\operatorname{diag}(1,1,1)$, and the coupling strengths are $c_{12}=c_{21}=\pi, c_{13}=c_{31}=\pi, c_{23}=c_{32}=\pi$, $c_{14}=c_{41}=2 \pi, c_{24}=c_{42}=2 \pi$, and $c_{34}=c_{43}=2 \pi$. Figure 3 visualizes this entire dynamical network.

The neural network is selected as

$$
\begin{gathered}
A_{n_{i}}=\left(\begin{array}{ccc}
-1 & 0 & 0 \\
0 & -1 & 0 \\
0 & 0 & -1
\end{array}\right), \quad W_{n_{i}}=\left(\begin{array}{ccc}
1 & 2 & 0 \\
-3 & 4 & 0 \\
0 & 2 & 3
\end{array}\right), \\
\sigma\left(x_{n_{i}}(t-\tau)\right)=\left(\begin{array}{c}
\tanh \left(x_{n_{i} 1}(t-\tau)\right) \\
\tanh \left(x_{n_{i}}(t-\tau)\right) \\
\tanh \left(x_{n_{i} 3}(t-\tau)\right)
\end{array}\right), \\
\tau=10 \text { seconds, } \\
L_{\phi_{\sigma_{i}}} \triangleq n=3, \\
x_{n_{i}}(0)=(20,20,-10)^{T}, \quad i=1,2,3,4 .
\end{gathered}
$$

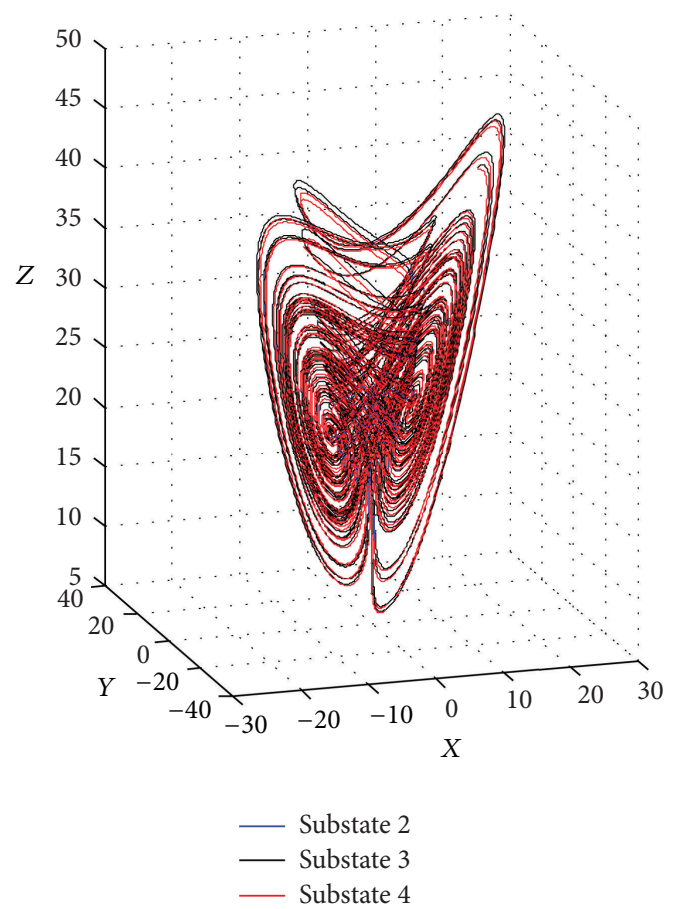

FIgURE 2: Sub-States of Chen's attractor with initial condition $X_{2,3,4}(0)=(-10 ; 0 ; 37)^{T}$.

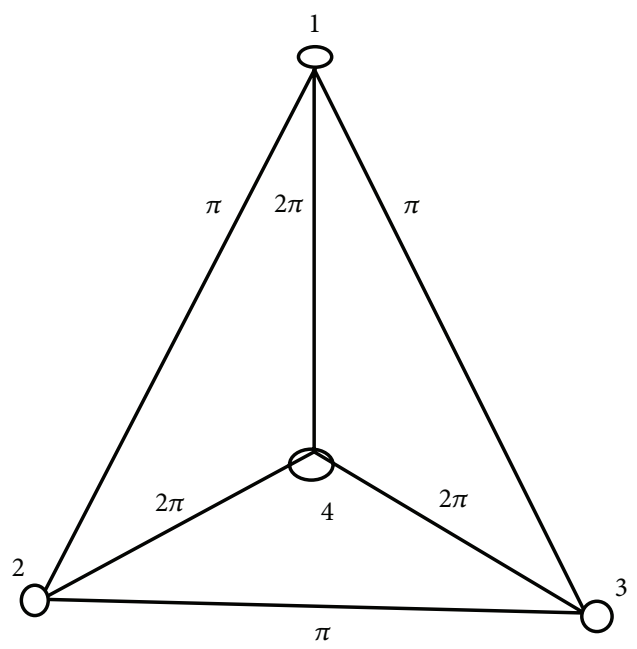

FIGURE 3: Structure of the network with each node being a Lorentz and Chen's system.

Theorem 2. For the unknown nonlinear system modeled by (1), the on-line learning law $\operatorname{tr}\left\{W^{T} W\right\}=-e^{T} W \sigma(x)$ and the control law (23) ensure the tracking of nonlinear reference model (4), [12].

Remark 3. From (21), we have $\dot{V}_{N}(e) \leq \sum_{i=1}^{N} e_{i}^{T}\left(-\lambda_{n_{i}} e_{i}-\right.$ $\sum_{\substack{j=1 \\ j \neq i}}^{N} c_{i j} a_{i j} \Gamma e_{i}+\left((1 / 2)+L_{\phi_{\sigma_{i}}}^{2}\left\|W_{n_{i}}\right\|^{2}\right) e_{i}+\widetilde{u}_{n_{i}}^{(1)}+\sum_{\substack{j=1 \\ j \neq i}}^{N} c_{i j} a_{i j} \Gamma e_{j}+$ $\left.\widetilde{u}_{n_{i}}^{(2)}\right)<0, \forall e \neq 0, \forall W$, and therefore $V$ is decreasing and 


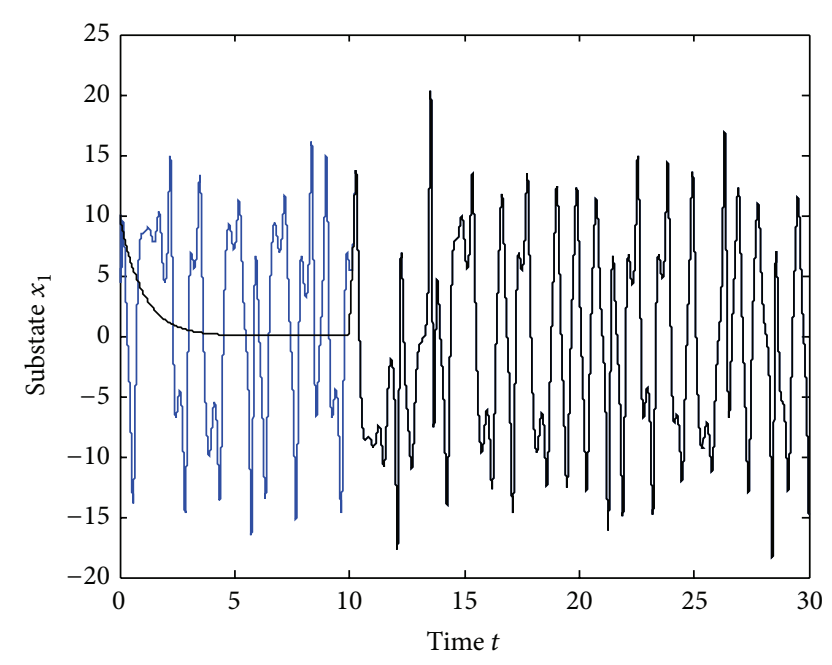

- Substate 1

— Neural network 1

FIgURE 4: Time evolution for sub-state 1 with initial state $X_{n 1}(0)=$ $(10 ; 0 ; 10)^{T}$.

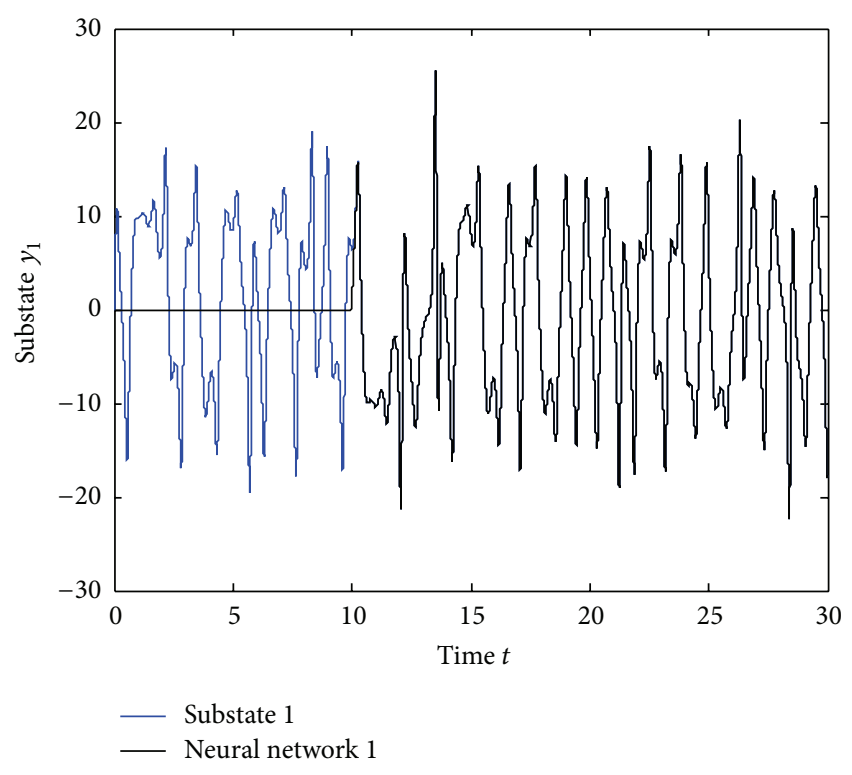

FIgURE 5: Time evolution for sub-state 1 with initial state $X_{n 1}(0)=$ $(10 ; 0 ; 10)^{T}$.

bounded from below by $V(0)$. Since $V_{N}(e)=\sum_{i=1}^{N}((1 /$ 2) $\left.\left\|e_{i}\right\|^{2}+\int_{t-\tau}^{t}\left(\phi_{\sigma}^{T}(s) W_{n_{i}}^{T} W_{n_{i}} \phi_{\sigma}(s)\right) d s\right)$, then we conclude that $e, W \in L_{1}$; this means that the weights remain bounded.

The experiment is performed as follows. Both systems, the delayed neural network (2) and the dynamical networks (24) and (25), evolve independently until $t=10$ seconds; at that time, the proposed control law (23) is incepted. Simulation results are presented in Figures 4, 5, and 6 for sub-sates of node 1 . As can be seen, tracking is successfully achieved and

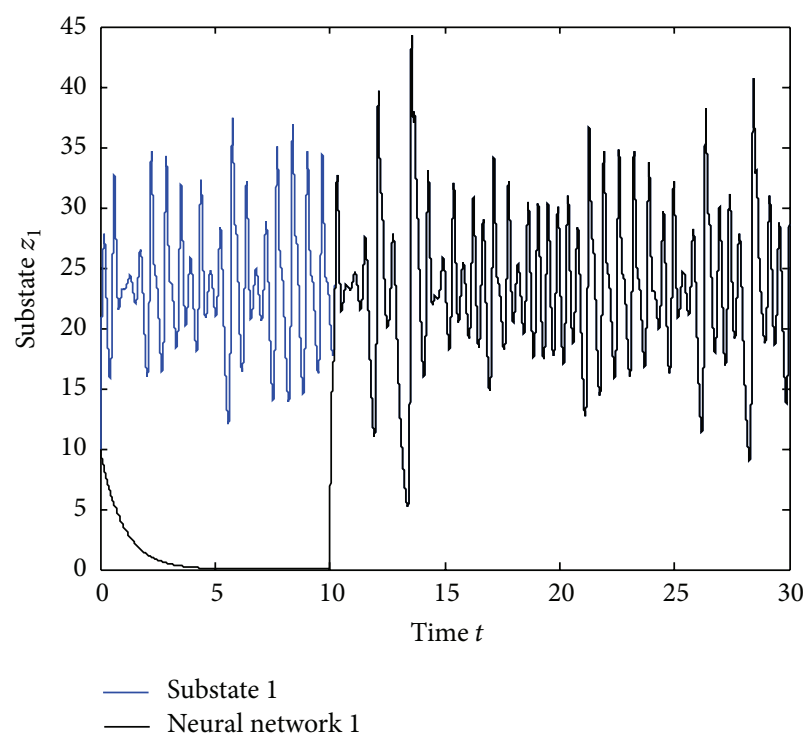

Figure 6: Time evolution for sub-state 2 with initial state $X_{n 1}(0)=$ $(10 ; 0 ; 10)^{T}$.

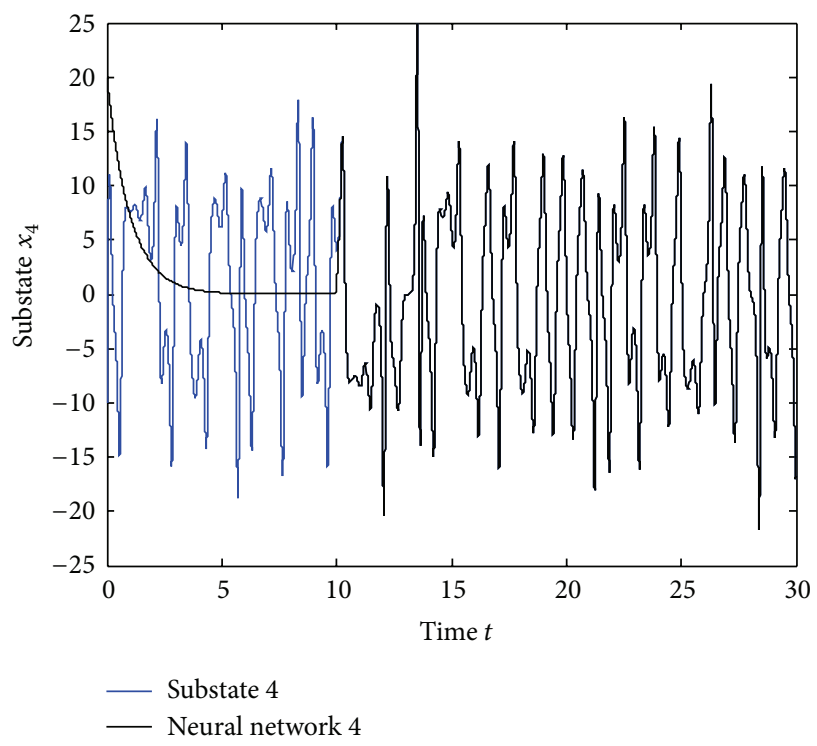

FIgURE 7: Time evolution for sub-state 4 with initial state $X_{n 4}(0)=$ $(20,20,-10)^{T}$.

error is asymptotically stable, as it is shown in Figures 7, 8, and 9 for sub-states of node 4 .

\section{Conclusions}

We have presented the controller design for trajectory tracking determined by a general complex dynamical network. This framework is based on dynamic delayed neural networks and the methodology is based on V-stability and Lyapunov theory. The proposed control is applied to a dynamical network with each node being a Lorenz and Chen's dynamical systems, respectively, being able to also stabilize in asymptotic 


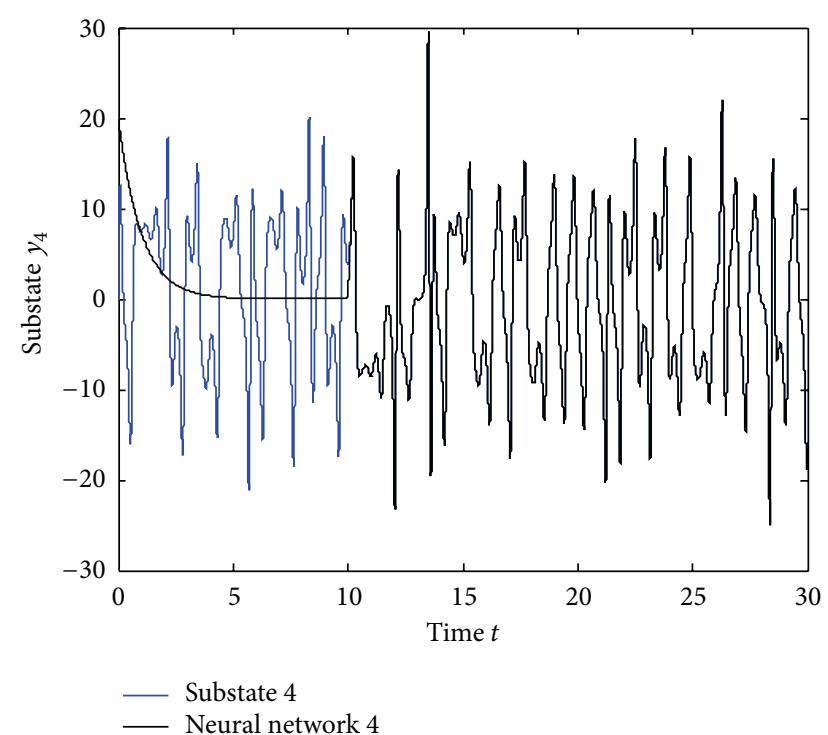

Figure 8: Time evolution for sub-state 4 with initial state $X_{n 4}(0)=$ $(20,20,-10)^{T}$.

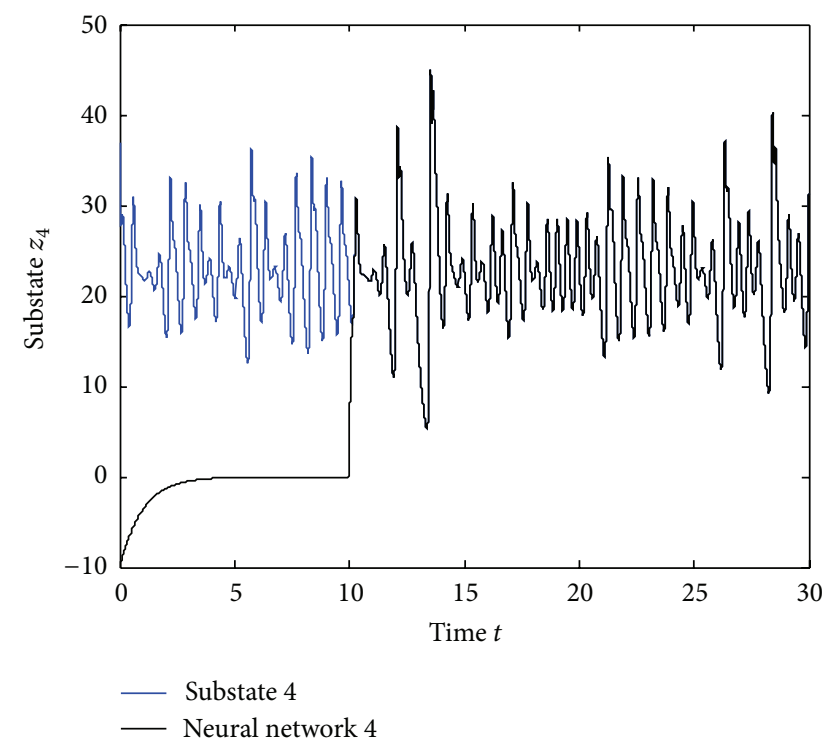

FIgURE 9: Time evolution for sub-state 4 with initial state $X_{n 4}(0)=$ $(20,20,-10)^{T}$.

form the tracking error between the two systems. The results of the simulation clearly show clearly the desired tracking. In future work, we will consider the stochastic case for the complex dynamical network.

\section{Conflict of Interests}

The authors declare that there is no conflict of interests regarding the publication of this paper.

\section{Acknowledgments}

The first and second authors thank the support of CONACYT and the Matematicas Aplicadas group of the Facultad de Ciencias Fisico-Matematicas, Universidad Autonoma de Nuevo Leon, Mexico.

\section{References}

[1] X. F. Wang, "Complex networks: topology, dynamics and synchronization," International Journal of Bifurcation and Chaos in Applied Sciences and Engineering, vol. 12, no. 5, pp. 885-916, 2002.

[2] C. Wu, Synchronization in Coupled Chaotic Circuits and Systems, World Scientific, Singapore, 2002.

[3] M. E. J. Newman, "The structure and function of complex networks," SIAM Review, vol. 45, no. 2, pp. 167-256, 2003.

[4] P. Baldi and A. F. Atiya, "How delays affect neural dynamics and learning," IEEE Transactions on Neural Networks, vol. 5, no. 4, pp. 612-621, 1994.

[5] X. Liao, G. Chen, and E. N. Sanchez, "LMI-based approach for asymptotically stability analysis of delayed neural networks," IEEE Transactions on Circuits and Systems I, vol. 49, no. 7, pp. 1033-1039, 2002.

[6] X. Liao, G. Chen, and E. N. Sanchez, "Delay-dependent exponential stability analysis of delayed neural networks: an LMI approach," Neural Networks, vol. 15, no. 7, pp. 855-866, 2002.

[7] H. Khalil, Nonlinear System Analysis, Prentice Hall, Upper Saddle River, NJ, USA, 2nd edition, 1996.

[8] M. Krstic and H. Deng, Stabilization of Nonlinear Uncertain Systems, Springer, New York, NY, USA, 1998.

[9] E. N. Sanchez and J. P. Perez, "Input-to-state stability (ISS) analysis for dynamic neural networks," IEEE Transactions on Circuits and Systems I, vol. 46, no. 11, pp. 1395-1398, 1999.

[10] E. N. Sanchez, J. P. Perez, and J. Perez, "Trajectory tracking for delayed recurrent neural networks," in Proceedings of the American Control Conference, pp. 2280-2284, Minneapolis, Minn, USA, June 2006.

[11] X. Li, X. Wang, and G. Chen, "Pinning a complex dynamical network to its equilibrium," IEEE Transactions on Circuits and Systems I, vol. 51, no. 10, pp. 2074-2087, 2004.

[12] P. A. Ioannou and J. Sun, Robust Adaptive Control, PrenticeHall, Upper Saddle River, NJ, USA, 1995. 


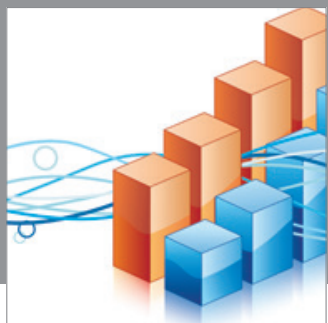

Advances in

Operations Research

mansans

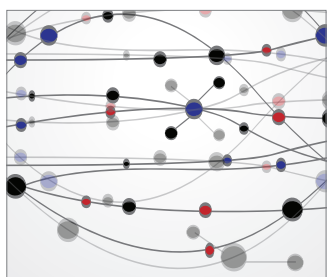

The Scientific World Journal
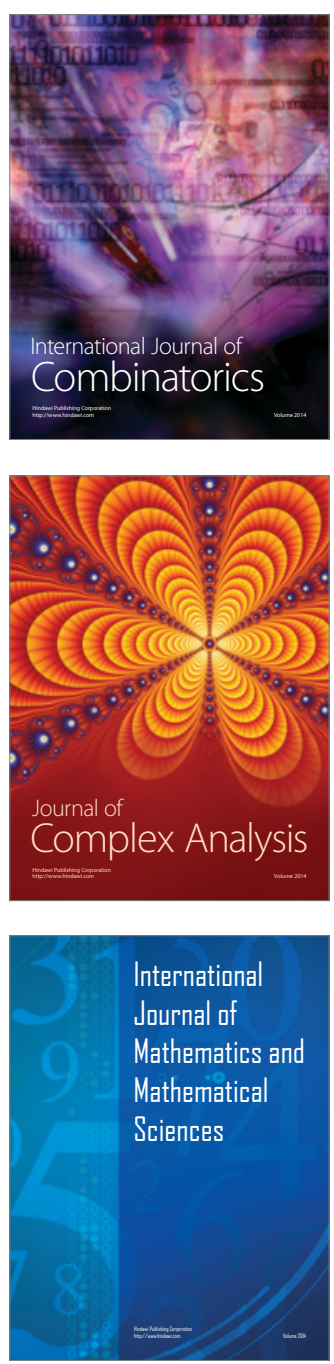
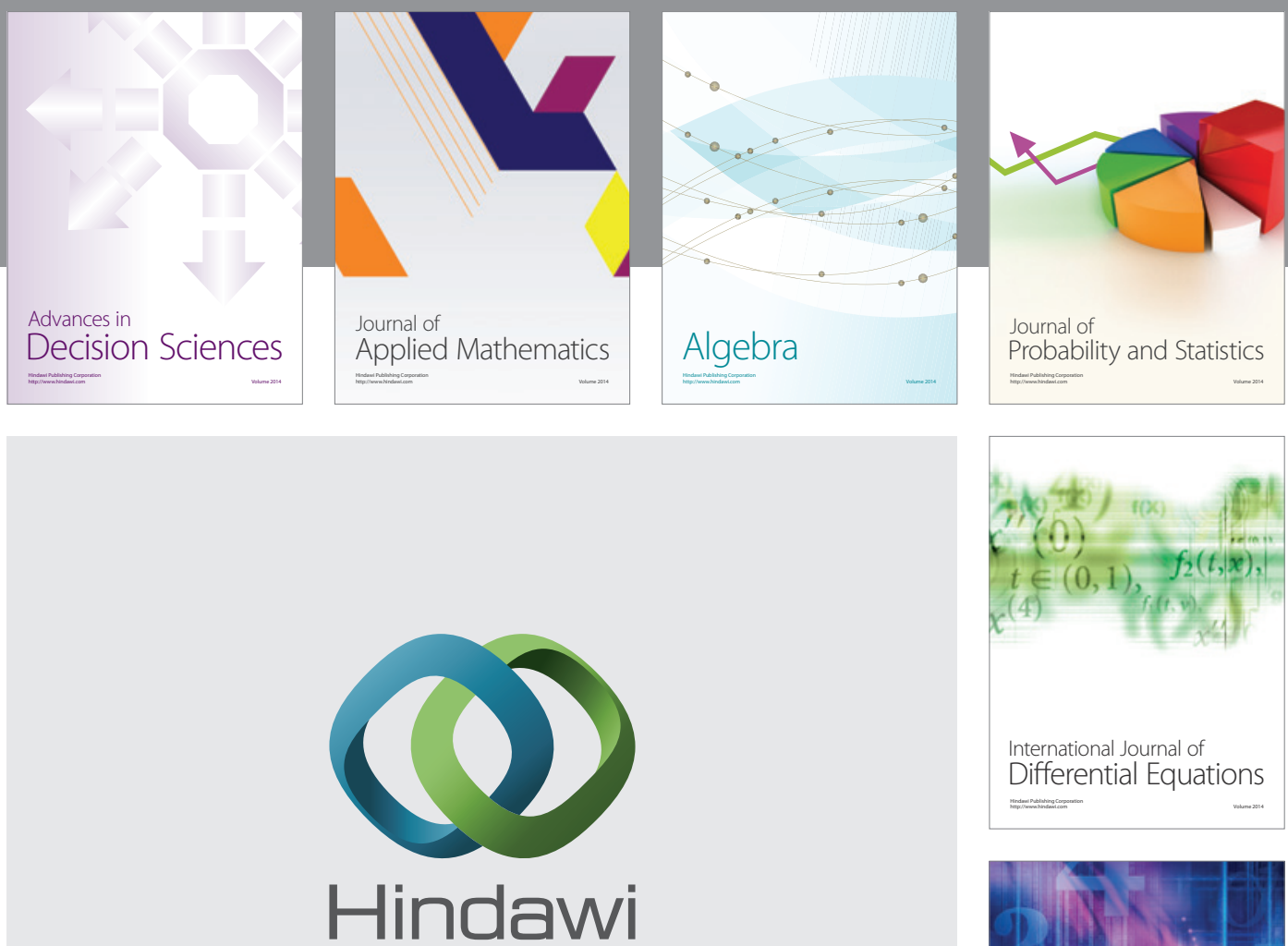

Submit your manuscripts at http://www.hindawi.com
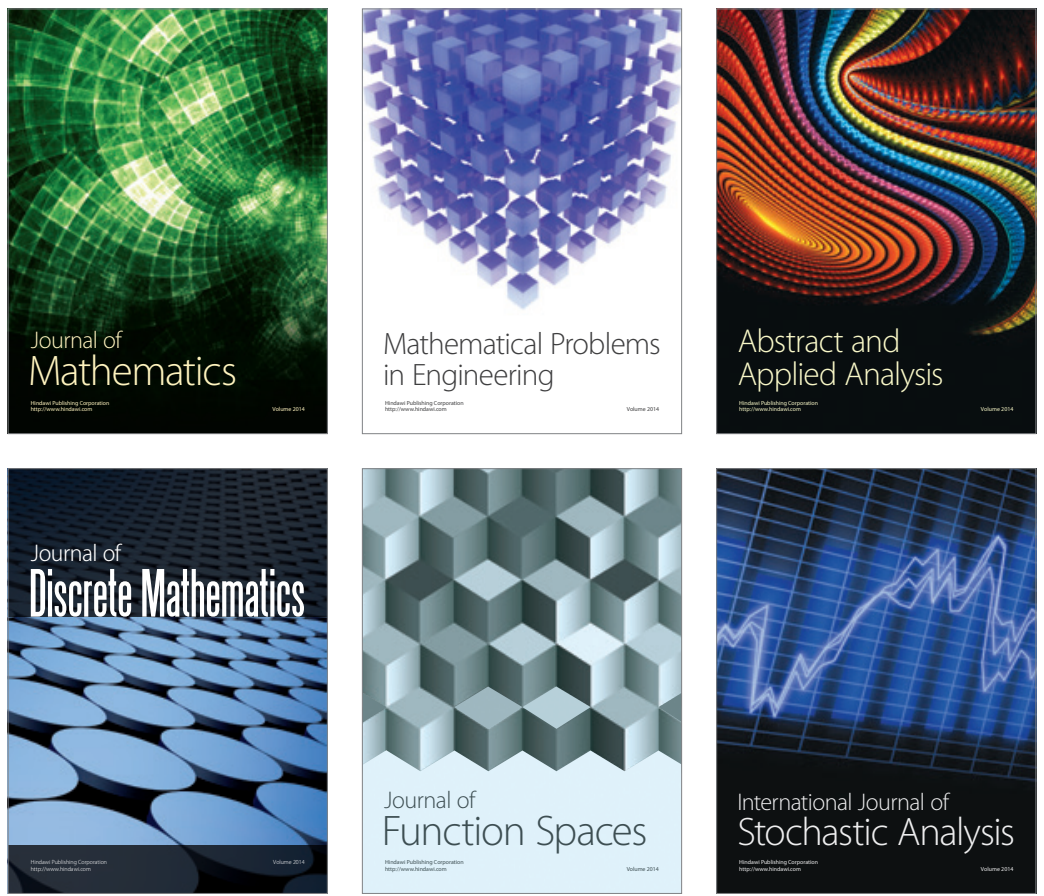

Journal of

Function Spaces

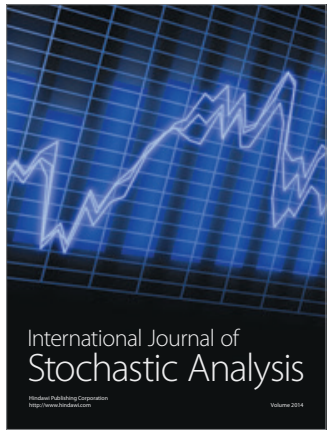

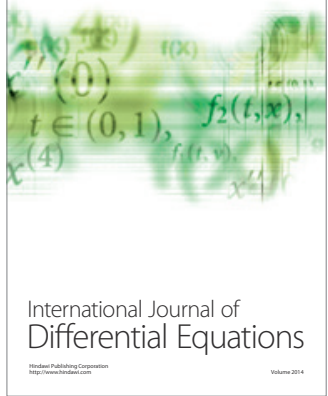
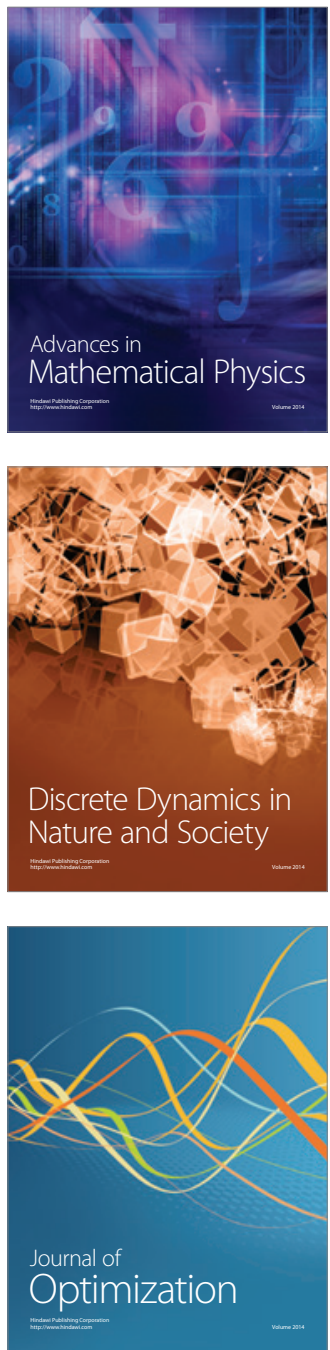